PROCEEDINGS OF THE

AMERICAN MATHEMATICAL SOCIETY

Volume 48, Number 1, March 1975

\title{
WHEN IS THE TENSOR PRODUCT OF ALGEBRAS LOCAL?
}

\author{
MOSS EISENBERG SWEEDLER I
}

ABSTRACT. Suppose the tensor product of two commutative algebras over a field is local. It is easily shown that each of the commutative algebras is local and that the tensor product of the residue fields is local. Moreover, one of the algebras must be algebraic over the ground field, i.e. contain no transcendentals. These three conditions characterize when the tensor product of commutative algebras is local.

Introduction. Throughout $A$ and $B$ are commutative algebras over a field $K$.

Theorem. (a) and (b) are equivalent:

(a) $A \otimes B$ is a local algebra.

(b) (i) $A$ is a local algebra (with maximal ideal $M$ ) and $B$ is a local algebra (with maximal ideal $N$ ).

(ii) Either $A$ or $B$ is algebraic over $K$, i.e. contains no transcendentals over $K$.

(iii) $A / M \otimes B / N$ is local.

That (b) implies (a) is easily seen as well as (a) implies (b, i) and (b, iii). The work lies in "(a) implies (b, ii)"'. This goes by means of

Lemma. If $a \in A$ and $b \in B$ are both transcendental over $K$, then $c=1 \otimes b$ $+a \otimes 1 \in A \otimes B$ is not invertible.

The Lemma is proved by means of the units theorem [S, Theorem 1.2, p. 260].

The Theorem of this paper reduces the question of when the tensor product of algebras is local to the question of when the tensor product of an algebraic field extension and an arbitrary field extension is local. We include a final proposition to further reduce the problem to the question of when the tensor product of a separable algebraic field extension and an arbitrary field extension is local and, hence, a field.

Received by the editors January 9, 1974.

AMS (MOS) subject classifications (1970). Primary 16A48, $13 \mathrm{H} 99$.

1Supported in part by NSF GP 25600. 
All tensor products are over $K$ unless indicated to the contrary.

Proof of Lemma. Let $\bar{K}$ be the algebraic closure of $K$ and $\bar{a}=a \otimes 1 \epsilon$ $A \otimes \bar{K}, \bar{b}=b \otimes 1 \in B \otimes \bar{K}, \bar{c}=1 \otimes b \otimes 1+a \otimes 1 \otimes 1 \in A \otimes B \otimes \bar{K}$. With the usual identification between $A \otimes B \otimes \bar{K}$ and $(A \otimes \bar{K}) \otimes_{\bar{K}}(B \otimes \bar{K})$, the element $\bar{c}$ corresponds to $1 \otimes \bar{b}+\bar{a} \otimes 1$. Moreover, $c$ invertible implies that $\bar{c}$ is invertible, and $a, b$ transcendental over $K$ implies that $\bar{a}, \bar{b}$ are transcendental over $\bar{K}$. Hence, we may assume $K$ is algebraically closed.

Let $S=\{P(a) \in A \mid 0 \neq P(X) \in K[X]\}$ a multiplicative system in $A$. If $a$ is transcendental, then $0 \notin S$, and an ideal $I$ is prime if it is maximal with respect to the property $I \cap S=\varnothing,[\mathrm{K}$, Theorem $1, \mathrm{p} .1]$. In this case $A / I$ is a domain, and if $a^{\prime}$ is the coset of $a$ in $A / I$, then $a^{\prime}$ is transcendental over $K$ by choice of $S$ and $I$. Similarly if $b$ is transcendental in $B$, there is an ideal $J$ where $B / J$ is a domain and the coset $b^{\prime}$ of $b$ in $B / J$ is transcendental. Moreover $1 \otimes b+a \otimes 1 \in A \otimes B$ invertible implies that $1 \otimes b^{\prime}+a^{\prime} \otimes$ $1 \in(A / I) \otimes(B / J)$ is invertible. Hence, we may assume that $A$ and $B$ are domains.

By [S, Lemma 1.1, p. 260] $K$ is algebraically closed in $A$ and $B$. If $a$ is transcendental over $K$ then $\{1, a\}$ is a $K$ linearly independent set. If $b$ is transcendental then $\{1, b\}$ is $K$ linearly independent and the tensor $1 \otimes b$ $+a \otimes 1$ has rank two; hence, it cannot be written in the form $x \otimes y, x \in A$, $y \in B$. By the units theorem [S, Theorem 1.2, p. 260], $1 \otimes b+a \otimes 1$ is not invertible. Q.E.D.

Proof of Theorem. (a) $\Rightarrow(\mathrm{b}, \mathrm{ii})$. Suppose $a \in A$ is transcendental over $K$. Let $b \in B$; if $1 \otimes b+a \otimes 1$ is not invertible in the local algebra $A \otimes B$, it must lie in the maximal ideal and $1 \otimes 1+1 \otimes b+a \otimes 1=1 \otimes b+(a+1) \otimes 1$ is invertible. The element $1+a \in A$ is also transcendental over $K$; hence, Lemma 1 applies to $1 \otimes b+a \otimes 1$ or $1 \otimes b+(a+1) \otimes 1$ to show that $b$ is algebraic over $K$.

(a) $\Rightarrow(\mathrm{b}, \mathrm{i})$. Say $\pi, \pi$ are distinct maximal ideals in $B$; then $\pi+\pi=$ $B$ and $A \otimes \mathbb{M}+A \otimes \Re=A \otimes B$. This contradicts $A \otimes B$ being local. Similarly $A$ must be local.

(a) $\Rightarrow$ (b, iii). $A \otimes B \rightarrow A / M \otimes B / N$ is a surjective algebra homomorphism.

(b) $\Rightarrow$ (a). Suppose $A$ is algebraic over $K$. By [J, Theorem 13, p. 308], the radical $M$ of $A$ is nil; hence, $M \otimes B$ is a nil ideal in $A \otimes B$ and lies in the radical of $A \otimes B$.

Since $A$ is algebraic over $K, A \otimes B$ is an integral extension of $K \otimes B$. Let $L$ be a maximal ideal of $A \otimes B$ and $\pi: A \otimes B \rightarrow(A \otimes B) / L$. Then $(A \otimes B) / L$ is a field which is integral over $\pi(K \otimes B)$ and, by $[\mathrm{K}$, Theorem 16 , 
p.11], $\pi(K \otimes B)$ is a field. Hence $L \cap(K \otimes B)$ is a maximal ideal which must be $K \otimes N$. Hence $L \supset A \otimes N$, and $A \otimes N$ lies in the radical of $A \otimes B$. Thus it suffices to prove that $(A \otimes B) /(M \otimes B+A \otimes N)$ is local. But the quotient algebra is isomorphic to $A / M \otimes B / N$. Q.E.D.

Proposition. Let $A$ be an algebraic field extension of $K$ with (unique) maximal separable subfield $S$, and let $B$ be an arbitrary field extension of K.

(a) If $A$ is a purely inseparable field extension of $K$, then $A \otimes B$ is local with nil radical.

(b) $A \otimes B$ is local if and only if $S \otimes B$ is local, in which case $S \otimes B$ is a field.

Proof. (a) Suppose $A$ is a purely inseparable extension and $K$ has positive characteristic $p$. If $x=\Sigma a_{i} \otimes b_{i} \in A \otimes B$ there is $n \in \mathbf{Z}$ with $a_{i}^{p^{n}} \in K$ each $i$. So $x^{p^{n}}=1 \otimes\left(\sum a_{i}^{p^{n}} b_{i}^{p^{n}}\right) \in K \otimes B$ a field. Thus every element of $A \otimes$ $B$ is nilpotent or invertible which proves (a).

(b) $A \otimes B=A \otimes_{S} S \otimes B$. Suppose $S \otimes B$ is local. $A$ is a purely inseparable extension of $S$, and so by part (a) the tensor product over $S$ of $A$ and the residue field of $S \otimes B$ are local. Hence, by the Theorem, $A \otimes_{S}(S \otimes B)$ is local. Conversely if $A \otimes_{S}(S \otimes B)$ is local, then, by the Theorem, $S \otimes B$ is local.

Now suppose $S \otimes B$ is local. By [A, Theorem I, p. 1] the radical of $S \otimes B$ is zero. Hence $S \otimes B$ is a field. Q.E.D.

\section{REFERENCES}

[A] S. A. Amitsur, The radical of a field extension, Bull. Research Council of Israel $7 \mathrm{~F}$ (1957), 1-10.

[J] N. Jacobson, The radical and semi-simplicity for arbitrary rings, Amer. J. Math. 67 (1945),300-320. MR 7, 2.

[K] I. Kaplansky, Commutative rings, Allyn and Bacon, Boston, Mass., 1970. MR 40 \# 7234.

[S] M. E. Sweedler, A units theorem applied to Hopf algebras and Amitsur cohomology, Amer. J. Math. 92 (1970), 259-271. MR 42 \#3154.

DEPARTMENT OF MATHEMATICS, CORNELL UNIVERSITY, ITHACA, NEW YORK 14850 